\title{
Research Hotspot Analysis of EPC Pattern Based on CiteSpace in China
}

\author{
Li Aimin ${ }^{1, a}$, Lu Jing 2 ,b \\ ${ }^{1}$ School of Management Engineering,Zhengzhou University,Zhengzhou, China \\ ${ }^{2}$ School of Management Engineering,Zhengzhou University,Zhengzhou, China
}

\begin{abstract}
In this paper, 385 articles from 2010 to 2020 in CNKI database are visualized and analyzed by using CiteSpace software, and corresponding knowledge maps are drawn to mine the literatures related to EPC, this paper describes the current research trends and hot spots in the field of EPC. It is found that the research on EPC mode management has received high attention. Meanwhile, the coordination and management between general contractors, subcontractors and owners, project management, international engineering and hydropower engineering are also the main research hotspots.
\end{abstract}

\section{INTRODUCTION}

The Engineering Procurement Construction (EPC) is a contract for the whole process or several stages of the design, procurement, construction and trial operation of a construction project entrusted by the owner. This model was introduced in China in the 1980s ${ }^{[1]}$. After 2000, the EPC pattern entered the rapid development stage, in recent years, with the "Belt and Road" initiative being proposed and gradually launched, China's EPC industry has been systematically and strategically promoted to achieve a more rapid development ${ }^{[2]}$.

In order to promote EPC mode, The Opinions on Promoting Sustainable and Healthy Development of Construction Industry issued by The State Council [2017] No. 19 clearly proposed to accelerate the implementation of general project contracting, and the general project contracting mode should be adopted for prefabricated buildings in principle ${ }^{[3]}$. Different from the traditional construction general contracting model, EPC contractors should be fully responsible for the construction requirements proposed by the whole owner, and the risk management content is broader and more demanding. Considering the risk identification and control from the perspective of the general contractor will have important theoretical significance and application value for the project risk management of the general contractor ${ }^{[4]}$.

Throughout the domestic literature on EPC model, most of them focus on a written summary of a project, mainly for qualitative research. At present, there are no scholars in China to measure and visualize the EPC pattern. Based on this, this paper uses the CiteSpace software to analyze the literature related to the EPC pattern in China web (CNKI) database, from the aspects of literature basic statistics analysis, key word collinearity analysis and key word cluster analysis, this paper makes visual data mining for the research literature of EPC pattern from 2010 to 2020, and sorts out the development context of EPC pattern research in China in the past ten years, this paper analyzes the current situation of EPC pattern, realizes the visualization of EPC pattern knowledge map, grasps the research hot spot and the development trend, provides the reference for the EPC pattern in-depth research in our country.

\section{RESEARCH DESIGN}

\subsection{Research Methodology}

Bibliometrics is a cross-science of quantitative analysis of all knowledge carriers by means of mathematics and statistics. CiteSpace is an information visualization software developed by Professor Chen Chaomei in The Java language in 2004. It focuses on the potential knowledge contained in scientific analysis and plays an important role in scientometrics, data and information visualization ${ }^{[5]}$. Since it was introduced into China in 2006, it has attracted extensive attention from domestic scholars. At present, CiteSpace software is widely used in the fields of natural science and social science.

Therefore, this paper combines the key analysis function of the software with the key research contents of the article. Firstly, the annual number of papers published in EPC research field, author cooperation and institutional cooperation are analyzed by data mining and bibliometric analysis, and then CiteSpace is used the software consists of three modules: keyword co-occurrence, keyword clustering and timezone view. It analyzes the research status, development process, hot topics and evolution trend of EPC field, hoping to provide reference for researchers in EPC field. 


\subsection{Data Source}

In this paper, using CiteSpace software EPC mode related papers in CNKI as the research sample, select "EPC" as a keyword to search, and with SCI source journals, EI source journals, core journals, CSSCI, CSCD as retrieval conditions, time chosen for the 2010-2020, preliminary search out with 885 references, one by one manually on the retrieval periodicals, rejecting international conference papers, reports, and research topics not related literature, eventually get effective related with 385 references.

\section{ANALYSIS OF BASIC CHARACTERISTICS OF EPC LITERATURE}

\subsection{Annual Trend of Literature}

Figure 1 is a line chart drawn according to the publication volume of EPC on Cn.COM from 2010 to 2020. It can be clearly and intuitively seen from the figure 1 that in the past decade, the literature published in EPC field showed a state of periodic fluctuation, while the literature published in the past two years showed a decrease phenomenon. On the whole, the number of researches in EPC field showed a trend of decrease, indicating that domestic researches on EPC had become increasingly mature.

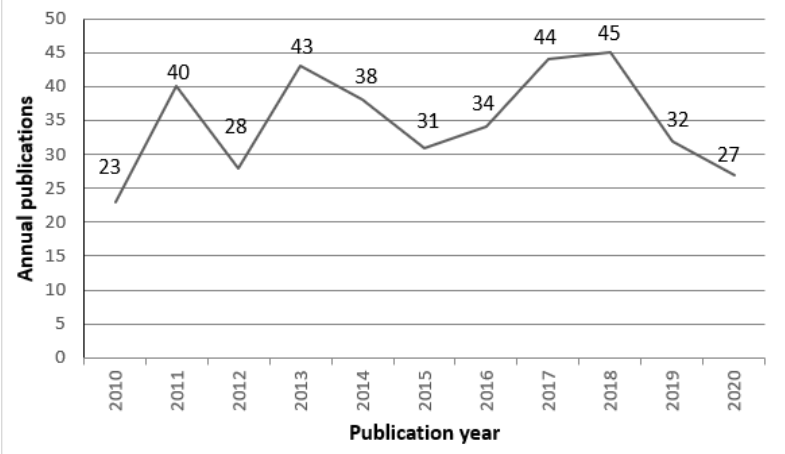

Figure 1. The quantity distribution of EPC research literature from 2010 to 2020

\subsection{Analysis of Authors and Publishers}

Import the literature in THE CNKI database into CiteSpace and convert it. The literature span is 2010.22020.8. Set Top $\mathrm{N}=50$, that is, take the keyword whose word frequency is listed in the first 50 of each event slice.

In order to systematically reveal the cooperation status between core authors and institutions, nodes in CiteSpace were set as "authors" and "institutions" respectively, and the co-occurrence knowledge maps of authors and research institutions as shown in Figure 2 and Figure 3 were obtained. The size and color spectrum of nodes represent the frequency and years of literature publication, respectively, and the thickness of the lines between nodes represents the closeness of cooperation between authors or institutions ${ }^{[6]}$.

The co-occurrence knowledge map of authors can identify the core group of authors in EPC research field, and the cooperative relationship between authors can also be clarified through the lines between nodes. As can be seen from figure 2 , the nodes shown in the map are $\mathrm{N}=52$, line $\mathrm{E}=39$, and the network density is 0.0294 , indicating that there are 52 people who have published more than 2 papers in EPC field. In terms of the frequency of authors, Wenzhe Tang published the most articles (10), followed by Zhang Hao (5), Zicheng Tao, Qingzhen Zhang, He Yanfang and Jianyun Yang, all of whom published 4 articles. It can be seen from this that the researches in EPC field are relatively scattered, and the core author group has not yet formed.

The co-occurrence knowledge map of research institutions can also clarify the core institutions in the EPC research field and the degree of close cooperation between them. Figure 3 has a total of 39 nodes and 6 links, that is, there are 39 scientific research institutions with more than 2 papers published. The longest generating paper was Yalong River Hydropower Development Co., LTD (7 papers), followed by The State Key Laboratory of Water and Sediment Science and Water Conservancy and Hydropower Engineering of Tsinghua University (5 papers each), East China Survey and Design Research Institute of Power Engineering Group of China and Iran Branch of China Petroleum Engineering Construction Corporation. In the figure 3 , most of the nodes exist in isolation except for a small number of nodes linked together, indicating that most of the current cooperation among institutions studying EPC is mostly concentrated in the same region, and there are few inter-regional cooperation exchanges. Moreover, most of the institutions are independent research, which may limit the innovation of EPC research to a certain extent.

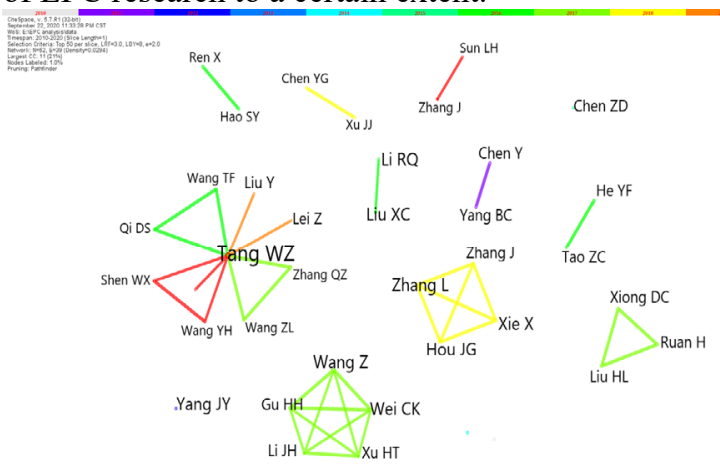

Figure 2. Author analysis

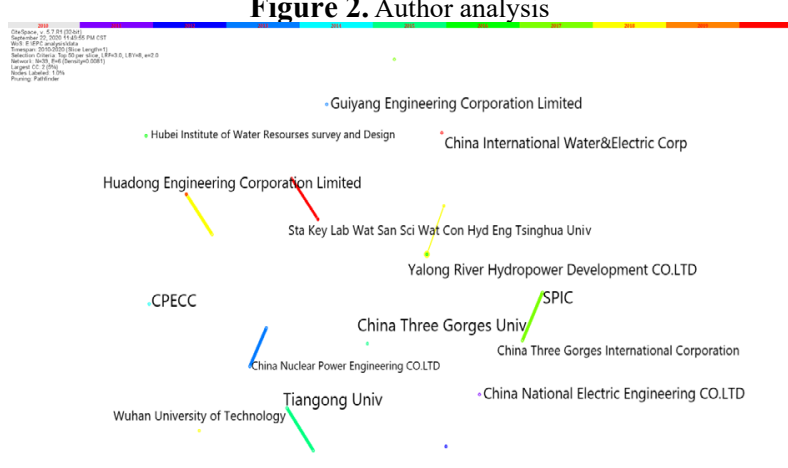

Figure 3. Analysis of issuing agencies 


\section{VISUAL ANALYSIS OF DOMESTIC EPC RESEARCH HOTSPOTS}

\subsection{Keywords Co-occurrence Analysis}

Set the nodes as Keywords in CiteSpace, and obtain the keyword co-occurrence knowledge map as shown in figure 4. Each node in figure 4 represents a keyword, and the size of the node represents the occurrence frequency of the keyword ${ }^{[7]}$. The lines in the figure 4 are crisscrossed and crisscrossed, indicating that the keywords are interrelated with each other. The thickness of the lines shows the strength of co-occurrence among the keywords. The depth of the lines indicates the time sequence of the keywords ${ }^{[8]}$. Can be seen from figure 4 , the graph shows the number of nodes $\mathrm{N}=70$, keyword connection between $\mathrm{E}=74$, network density is 0.0306 , explains the key word co-occurrence condensation degree is low, in addition, it can be seen in figure 4 , by CNKI database frequency is higher in the study of EPC in keywords have EPC project, EPC general contracting, EPC, general contractor, project management, international engineering, general contractor, management mode, etc., to reflect the current hot research topic in the field of EPC.

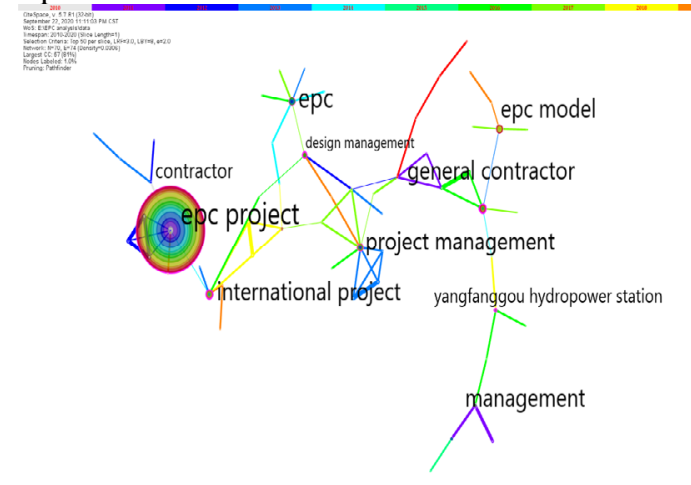

Figure 4. Keywords co-occurrence knowledge map

\subsection{Keyword Cluster Analysis}

LLR algorithm was used to conduct keyword clustering analysis of selected literatures in this paper, and the map of keyword clustering knowledge was obtained as shown in figure 5. The Modularity $\mathrm{Q}=0.7511>0.3$ and the Mean contour coefficient of clustering Mean Silhouette $=0.6483>0.5$, indicating that the structure of clustering is significant and highly reasonable. According to the clustering results, domestic EPC research is mainly divided into 7 topics, representing 7 main directions of EPC research in China, namely, \#0 "risk sharing", \#1 "general contracting", \#2 "Localization strategy", \#3 "management", \#4 "Pakistan", \#5 "EPC general contracting" and \#6 "System dynamics".

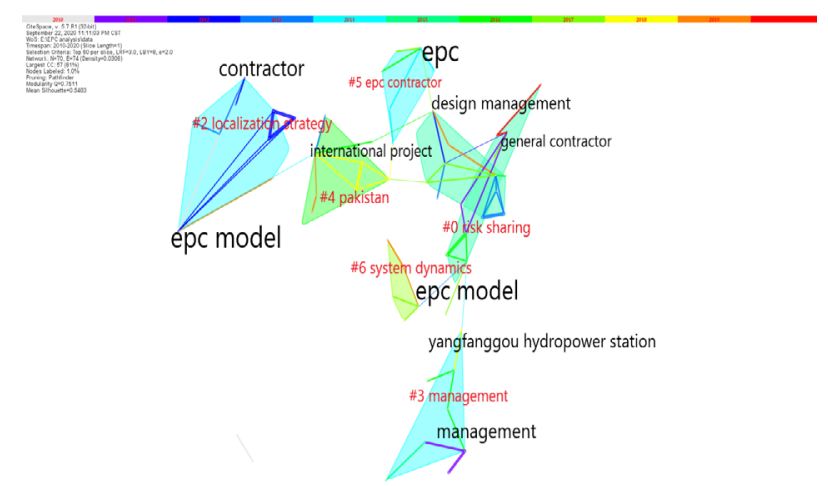

Figure 5. Keywords clustering knowledge map

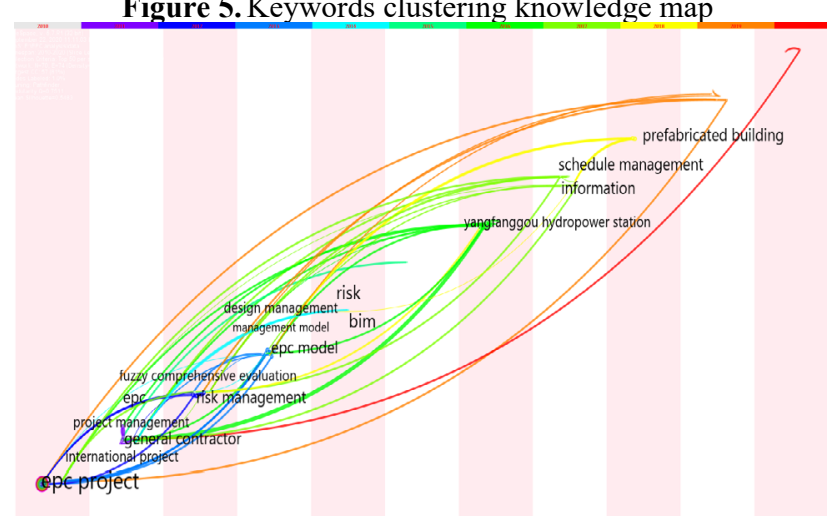

Figure 6. Evolutionary analysis of research

\subsection{Evolutionary Analysis of Research}

In order to more intuitively reflect the evolution process of research hotspots in EPC field, this study also USES the Timezone View function in CiteSpace software to display the clustering results according to the timeline View, as shown in Figure 6. According to the timeline view, we can analyze when each cluster begins to appear, when it rises, and when it declines, and further analyze the temporal characteristics of the research field reflected by each cluster. Nearly 10 years, management research of the EPC patterns dominate, most authors try to explore from the management perspective of issues related to the field [9], such as design and management, risk management, safety management, human resource management, quality management, contract management, knowledge management, etc., among them, because most of the EPC project is relatively complex, its risk management has always been the hot spot of the research in this field, at the same time, the current study, for EPC will be combined with the specific analysis of the EPC project problems of EPC mode, and put forward the corresponding countermeasures.

\section{CONCLUSION}

In this paper, literature on EPC research from 2010 to 2020 was collected through CNKI database, and visual analysis was carried out using CiteSpace software. Literature distribution map and co-occurrence knowledge map of authors and institutions were drawn to study publication characteristics and other aspects. From the cooccurrence analysis results of authors and research institutions, it can be seen that authors in EPC research 
field are relatively dispersed, research institutions are mainly enterprises and universities, and cooperation between institutions mostly occurs in the same region, so cross-regional research can be realized in the future. By drawing the key word co-occurrence and clustering knowledge map and time line view, combining the analysis results and the practice of EPC field in recent years, it shows that the overall research perspective of EPC field in China is relatively extensive and scattered, and some core research contents are relatively concentrated. Research hotspots mainly focus on EPC mode management, including coordination and management between general contractors, subcontractors and owners, project management, international engineering, hydropower engineering and so on.

To sum up, domestic scholars have carried out indepth studies on EPC mode and made certain scientific achievements. This paper clarified the current research hotspots of EPC mode by sorting out the development context of EPC mode in China in the past ten years, which can provide reference for relevant scholars in this field to some extent.

Finally, the following Suggestions are proposed for the future study of EPC mode:

(1) At present, research methods in EPC field are relatively concentrated, most of which adopt the fuzzy comprehensive evaluation method. Therefore, the diversity of research methods should be enhanced to combine the qualitative method and the quantitative method, so as to give full play to their advantages.

(2) The previous literature samples are mainly academic theoretical literature, and policy literature on EPC mode accounts for a very small proportion. However, both theoretical literature and practice reflect that national policies and regulations in this field need to be further improved in order to enhance their comprehensive universality. Therefore, it is necessary to strengthen the analysis and research of policy literature, and the comparative analysis between these two types of literature is also worth in-depth study.

(3) Strengthen the exchange and cooperation between research institutions and authors. From the writer's cooperation and research institutions can be seen in the knowledge map, the current domestic EPC research is mostly independently, to a certain extent hindered the development of research, nowadays, scientific research cooperation becoming the mainstream way of research, it is able to put our heads together, increase the diversity, cultivating innovative talents, therefore, scholars in the field of EPC, develop extensive cooperation with research institutions should strengthen the depth of the research, and promote innovation ability and scientific research level, provide strong support for the further development of EPC mode.

\section{REFERENCES}

1. Li Huiling, Mou Yonglin. Research on risk management of EPC project [J]. Construction economics,2020,41(S1):103-107.

2. Tan Zhongjie. Research on the Development Environment and Suggestions of EPC Mode in China [J]. Construction Economy, 2018,39(11):5-7.

3. Ji Yingbo, Yao Fuyi, Tong Wenjing, Zhang Qi. Design and Application of performance Evaluation Index System for EPC fabricated construction enterprises [J]. Science and Technology Management Research, 2018,38(11):188-194.

4. Ma Liang, Xiao Yi, Chen Guodong, Zhu Jun. A review of research literature in the field of general engineering contracting in China [J]. Journal of civil engineering and management,2019,36(01):83-89.

5. Chen Yue, Chen Chaomei, Liu Zeyuan, Hu Zhigang,, Wang Xianwen. Methodological function of CiteSpace knowledge Atlas [J]. Scientific research,2015,33(02):242-253.

6. Chen Jie, Chen Chaomei. CiteSpace: Text Mining and Visualization in Technology (2nd edition) [M]. Beijing: Capital University of Economics and Business Press,2017.

7. Bao Huimin, Sun Jian. Research overview of Citespace-based big data technology in engineering management field $[\mathrm{J}]$. Journal of civil engineering and management,2020,37(04):131-137.

8. Wang Tiegang. Effect evaluation and countermeasures of EPC general contracting model [J]. Journal of huaqiao university (natural science edition),2017,38(02):169-174.

9. Jiang Zuobin. Some Issues and Suggestions on EPC Project Implementation process [J]. International Economic Cooperation,2018(11):88-91. 